\title{
The Application of FDS used in the Cabin Fire Simulation and Human Evacuation of Civil Aviation
}

\author{
Zhi-jing Yu \\ Aviation Ground Special Equipments \\ Research Base \\ Civil Aviation University of China \\ Tianjin, China \\ iahilub@163.com
}

\author{
Qiang Xu \\ Aviation Ground Special Equipments \\ Research Base \\ Civil Aviation University of China \\ Tianjin ,China \\ iahilub@163.com
}

\author{
Jun-wei Han \\ Aviation Ground Special Equipments \\ Research Base \\ Civil Aviation University of China \\ Tianjin ,China \\ iahilub@163.com
}

\begin{abstract}
Considering the characteristics of fire in the cabin of Civil Aviation, a new method is introduced to do the fire simulation by using computer simulation. Some basic theories, computational models and practical problems are given about the fire dynamics simulation software FDS and evacuation software Evac. Finally, an example of cabin fire is used to explain how to employ the software and apply the results to the practical evacuation and rescue of cabin fire.
\end{abstract}

Keywords- cabin fire; fire simulation; human evacuation; FDS ; Evac

\section{INTRODUCTION}

Modern civil aircraft cabin is luxuriously decorated, fully equipped. But these materials are mostly combustible items. Decorative materials, seats, carpets, and passengers' luggage within the cabin are burning fire load. On the other hand the cabin space is relatively small with large number of people, so the possibility of resulting in serious accidents because of the electrical fault or man-made causes is good. For example, on December 24th, 1982, an Il-18 202 aircraft was in fire by the combustion of combustible materials in the cabin floor because of a cigarette butt. The fire was found in smoke at 17:18, and three minutes later the aircraft landed in Guangzhou Baiyun airport. When the 11 passengers evacuated, the smoke of the cabin thickened. Because of the small evacuation routes and confusion of the travelers, 25 people of 69 passengers died and 26 others injured. So it is very necessary to study the fire inside the cabin and human evacuation. However, this type of fire is a small probability event, and the simulation study using experimental methods will have significant limitations because of the cost and other reasons, therefore we can consider computer simulation methods ${ }^{[1]}$.

\section{THE INTRODUCTION TO SOFTWARE OF FDS AND EVAC}

\section{A. FDS}

FDS (Fire Dynamic Simulator) is a Computational Fluid Dynamics (CFD) software mainly for the study of fluid movement in the fire. It is developed by the U.S. National Institute of Standards and Technology (NIST). Because FDS program is open, and its accuracy has also been verified by a large number of large-scale and full-scale fire experiments. So the FDS software has been widely applied in the field of fire science. The FDS software is mainly used in building fire simulation, but it can also be used for fire simulation in the other objects, such as transport trains, ships, tunnels, mines, etc. But its application in the aircraft is rare, so in this article the theoretical basis of FDS, tips and specific issues that should be noted in the cabin fire simulation are analysed.

FDS 5.0 version integrates FDS fire simulation software, the Evac fire evacuation simulation software and the SmokeView of visualization software. Evac is a human evacuation simulation software developed by the Technical Research Centre of Finland (VTT) which is based on a model of continuous space. When used together with the FDS software, it can simulate the real fire evacuation scenarios, and the simulation results could be displayed in the form of animation by the visualization software SmokeView.

1) Mathematical model

Some basic equations for FDS using large eddy simulation method to calculate are as follows ${ }^{[2]}$ :

Continuity equation:

$$
\frac{\partial \dot{u}}{\partial t}+\nabla \bullet \stackrel{r}{r}=0
$$

Momentum conservation equation:

$$
\rho\left(\frac{\partial u}{\partial t}+\frac{1}{2} \bullet \nabla\left|\begin{array}{c}
\mathrm{r} \\
u^{2}
\end{array}\right|^{\mathrm{r}}-\stackrel{\mathrm{u}}{u} \times \omega\right)+\nabla p-\rho g=\stackrel{\mathbf{u}}{f}+\nabla \bullet \sigma
$$

Energy conservation equation:

$$
\begin{aligned}
& \frac{\partial}{\partial t}(\rho h)+\nabla \bullet(\rho \stackrel{r}{u})= \\
& \frac{\partial p}{\partial t}+\stackrel{r}{u} \bullet \nabla p-\nabla \stackrel{r}{q} r+\nabla \bullet(k \nabla T)+\sum_{i}\left(h_{i} \rho D_{i} Y_{i}\right)
\end{aligned}
$$

Component conservation equation:

$$
\frac{\partial}{\partial t}\left(\rho Y_{i}\right)+\nabla \bullet\left(\rho Y_{i}^{r} u\right)=\nabla \bullet\left(\rho D_{i} \nabla Y_{i}\right)+m_{i}^{\prime \prime \prime}
$$

Gas state equation: 


$$
p_{0}=\rho T R \sum_{i}\left(Y_{i} / M_{i}\right)
$$

In the equations (1)-( 5 ) : $\rho$, Density, Unit: $\mathrm{kg} / \mathrm{m}^{3} ; \dot{u}$, Velocity vector, Unit: $\mathrm{m} / \mathrm{s}$; p,Pressure, Unit: $\mathrm{Pa}$; h, Total enthalpy,Unit: J/kg; T,Temperture, Unit:K; $Y_{i}$,Single mass concentration of component i; $D_{i}$, The diffusion coefficient for component I, Unit: $\mathrm{m}^{2} / \mathrm{s} ; M_{i}$, Molar of component I, Unit:kg/mol; $W_{i}$, The chemical reaction rate of the component I, Unit:kg/( $\left.m^{3} \mathrm{~s}\right) ; \sigma$, Viscous stress tensor, Unit:Pa ; q, Combustion heat of reaction, Unit: $\mathrm{J} / \mathrm{mol}$; $\mathrm{k}$, the thermal conductivity, Unit:W/(mK); g,Gravity vector; t,Time, Unit: s.

\section{2)The Application Process of FDS}

As shown in Figure 1, before the FDS simulation, you should better have a deep understanding about the simulation objects and get a full access to its basic conditions, such as size, material, structure, etc., and only accurate information can ensure the accuracy of the simulation results.

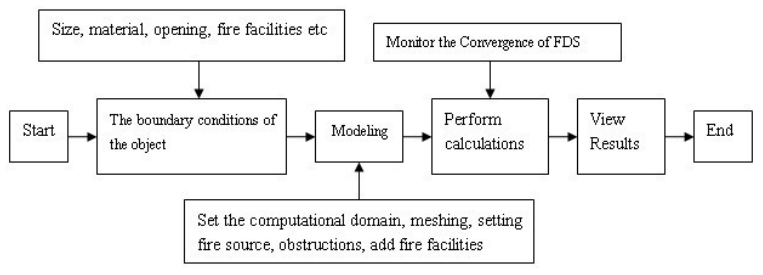

Figure 1. The Application Process of FDS

When establish the FDS model, It should be noted that FDS only support rectangular form of the calculation model ${ }^{[3]}$ and other shapes should be modeled with small rectangular approximation .So for the complex shape model, calculation deviations may occur and calculation amount increases. Then in actual applications, the FDS is mainly used to calculate the models of rectangular style, and some approximate rectangular model can be simplified into rectangular models. Although there are a certain deviations but the structure is simple to calculate and the time shorten, so the simulation results are still very valuable. The example in this article, we can simplify the cabin into a rectangular style for simulation.

When meshing, the grid unit should be as close as possible to the shape of cube, and this will help to improve the accuracy of the calculation results. Also note that a large parts of FDS calculation use the method of Poisson Distribution (Poisson solver) based on Fourier rapid conversion formula (FFTs), so the grid unit size should try to meet this modulus $2^{l} 3^{m} 5^{n}, 1, m, n$ are integers. For example, $72=2^{3} 3^{2}$, $108=2^{2} 3^{3}$, so 72,108 are good mesh cell sizes, and $37,99,109$ are not good sizes ${ }^{[4]}$.

There are two aspects when set the fire source. One is the location of the fire source, and the other is the size of the fire source. There are two types to the size of the fire source. One is the fixed Heat Release Rate (HRR) of the fire source, and the other is a heat release rate changing with time. The former one is simple and convenient, but the latter which is closer to the true fire scene.

\section{$B$ Theoretical basis of Evac}

1) The physical characteristics model of evacuation person

In the Evac software, evacuation person's body model is a three circle model. As shown in Figure 2, three overlapping circles represent the physical characteristics of the human body.

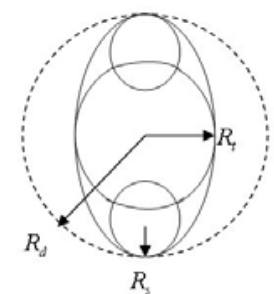

Figure 2. The physical characteristics model of evacuation person

The great circle in the middle represents body round, and the two small circles on both sides represent arm round, while the outside oval represents the projection of the human body in the ground. $R_{t}$ is the radius of the circle of the human body . $R_{d}$ is the size from the center of the body to the outermost end of shoulders, and $R_{\mathrm{s}}$ is the radius of the shoulder circle. It also defines five types of personnel (adult, men, women, children, the elderly), and they all correspond to different body size parameters and walking speed. Note that the default software setting is the human dimension of the Europe and the United States, while the body sizes of the Chinese people have a greater deviation. Because the new Chinese human body size table has not been announced, so it can be set in accordance with the GB10000-1988 standard.

\section{2) The model of fire injury to the human body}

Body injury model is a model proposed by NIST as N-gas. It assumes that most of the fire burning toxicities are a few $\mathrm{N}$ gases. But current vision of FDS only consider the Stifling effect of $\mathrm{CO}_{2}$, The stifling effect of hypoxia and the Toxic effect of $C O$.It describes the size of the overall toxicity with FED (FED: Fractional Effective Dose) to describe. The following are the FED formulas ${ }^{[5]}$ :

$$
\begin{aligned}
F E D_{\text {tot }} & =F E D_{c o} \times H V_{c o_{2}}+F E D_{o_{2}} \\
F E D_{c o} & =4.607 \times 10^{-7}\left(C_{c o}\right)^{1.036} t \\
F E D_{o_{2}} & =\frac{t}{60 \exp \left[8.13-0.54\left(20.9-C_{o_{2}}\right)\right]} \\
H V_{c o_{2}} & =\frac{\exp \left(0.1930 C_{c o_{2}}+2.0004\right)}{7.1}
\end{aligned}
$$

In (6) -(9) , FED tot - The FED value of fire smoke; $F E D_{c o}$ - the FED value of $\mathrm{CO} ; \mathrm{HV}_{\mathrm{Co}_{2}}$ - The choking effect impact factor of $\mathrm{CO}_{2} ; \mathrm{FED}_{\mathrm{o}_{2}}$ - The FED value of 
hypoxia suffocation; $C_{c o}$-The concentration of $C O$; $\mathrm{C}_{\mathrm{CO}_{2}}$ - The concentration of $\mathrm{CO}_{2}$.

\section{SET UP THE CABIN FIRE MODEL AND SIMULATION APPLICATIONS}

A320 series aircraft developed by Airbus Industry is a single channel and electric control civil aircraft, and it is the world's first civil airliner of electric control and fly-by-side rod driving with twin-engine, subsonic, and applicable in short lines. The A320 has been the main model of Chinese Airlines. At the end of March 2012, there are a total of 16 airlines with 666 Airbus A320 series aircraft, accounting for $40 \%$ of the 100 -seat level aircrafts in China. So this paper chooses the A320 cabin as the fire simulation object.

FDS modeling approach is achieved through the text program and it does not have the function of visual modeling. This requires the user have a very strong imagination of space and the ability to handle a large number of precise coordinates, which caus great inconvenience to the user. To solve this problem, the NIST recommend several visualization modeling softwares as the pre-processor of the FDS. Among them, PyroSim produced by the Thunderhead Engineering Company is a good pre-processing modeling software, so this article use PyroSim as the software to set up the model.

\section{A. The simulation boundary conditions of the cabinet}

The cabin is located in a sealed space between the cockpit and the rear of the aircraft .The equipments and facilities in the cabinet include the passenger seat, kitchen and toilet and so on Refer to the manual of Airbus A320, we can get these cabin sizes: a length of $27.5 \mathrm{~m}$, a width of 3.7 meters and a height of 2.2 meters. There are 4 doors in the front and behind of the cabin , 4 emergency exit doors, and two toilets. In accordance with the terms of the FAR25.853 (American Airlines Management coherent - transport category airplanes - cabin internal implementation), it shows that the fixed fire loads in the aircraft cabin are seats, carpet, decorations, as well as the mobile fire load - passenger luggage.

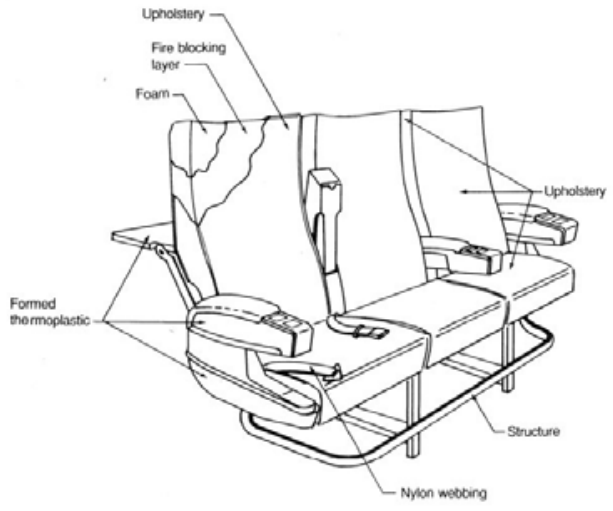

Figure 3. The material of the passenger seat

As shown in Figure 3 is the material of the seat on the aircraft. We can see that foam (Foam), thermal plastic (Thermoplastic), decorative materials (Upholstery) is easy to burn, so we can set these three types of materials as the combustion of the cabin fire simulation. We set the indoor and outdoor initial average temperature of 20 degrees Celsius as ambient temperature, the relative pressure as 0 (local atmospheric pressure $\mathrm{P}=101,325 \mathrm{~Pa}$ ).

\section{B. Set up the cabin model}

As shown in Figure 4, we set up the cabin model in accordance with the real size of the A320 cabin, with the X, Y-direction mesh size of $0.1 \mathrm{~m}$, and $0.09 \mathrm{~m}$ of the Z-direction. There are total 24,3455 grid units in this model. Because Evac software's minimum prevailing dimensions is 0.7 meters, which means that if the size of the space between the obstacles is less than 0.7 meters, the evacuation person will not be able to pass. So we simplify the typical 150 -seat cabin layout of A320 into 18 rows, each row of six seats, total of 128 seats. Then the evacuation person can move from his seat to the aisle. There are intake ports and exhaust ports on the top of the cabin. We set the thermocouples and smoke detectors in different locations of the cabin to detect the temperature and smoke concentration of each location.

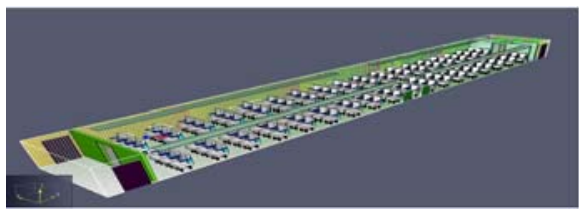

Figure 4. The overall model of the cabin

As shown in the figure 4, the Fixed Heat Release Rate(HRR)fire source is set at the middle seat of the second row. As shown in the figure 5 , the main obstacles are as follows: Seats (including the cushion, backrest, armrest, pallets, etc.), the walls of the toilet, luggage rack, carpet, etc.

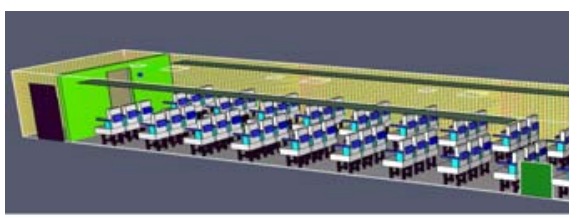

Figure 5. The local figure of the cabin model

\section{Set up the model of evacuation person}

There are random distributions of the four types of evacuation persons in the cabin, respectively men, women, children, the elderly, with 30 persons of each type. Their reaction time, body size, walking speed and other parameters can be adjusted accordingly, and it should be noted that the initial density of the staff should be less than four people per square meter, otherwise FDS will prompt an error in the simulation.

\section{The results and applications of the simulation}

We can do the fire simulation first .If there is no error, then do the evacuation simulation, which is easy to find errors.

You can view the spread of fire as well as evacuation by SmokeView software. As shown in Figure 6-9, The fire gradually spread throughout the cabin, and persons are also beginning to escape 


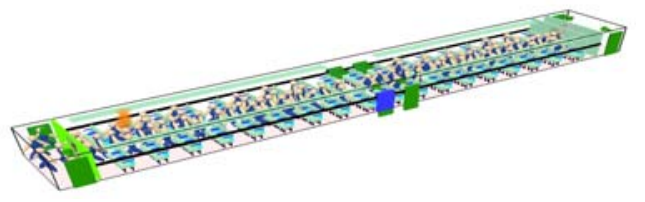

Figure 6. The fire begin to develop

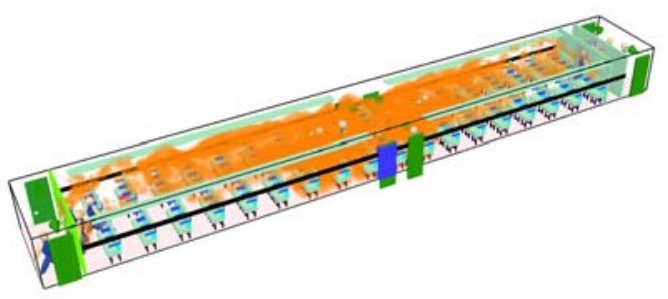

Figure 7. The cabin is all in the fire

As shown in Figure 8, the smoke first rose to the ceiling and then backward. After filled the entire cabin, it began to sink down.

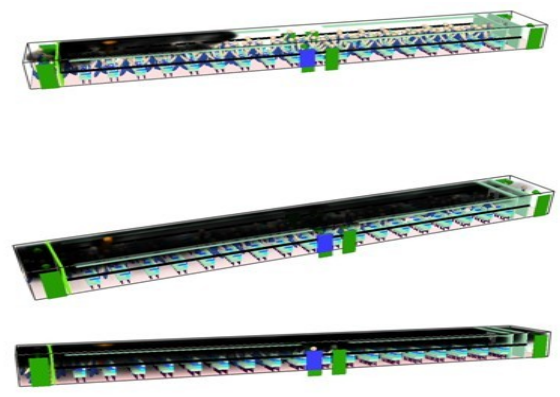

Figure 8 . The spread of the smoke in the cabin

As shown in Figure 9-12, in addition to the intuitive view of fire development by the SmokeView software, other fire data can be displayed by FDS software to view the curve line. As shown in Figure 12, there is a sudden temperature surge in 80 seconds. A flashover occurs, and the whole cabin is in the fire. It can be confirmed by the Figure 8 . Before the 80 second, there is no one died, but after that some people died. From Figure 10 evacuation curve, we can see that the majority of people escaped before the fire flashover in 80 seconds, and the rest were difficult to escape.

AllAgents

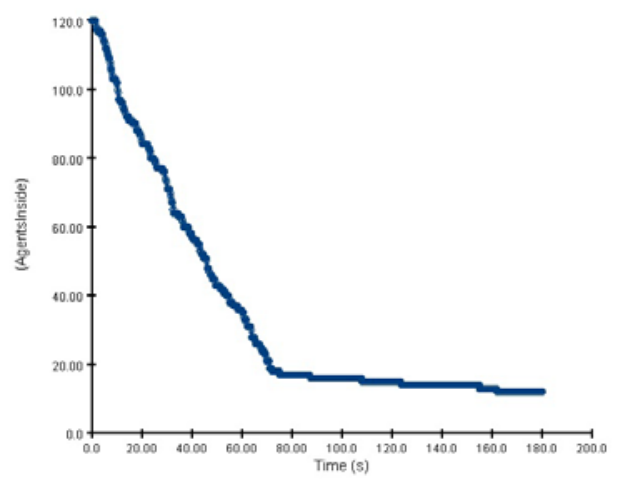

Figure 9. The curve of evacuation

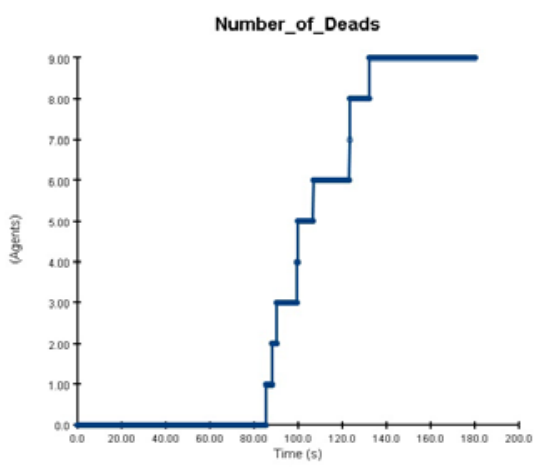

Figure 10. The curve of death

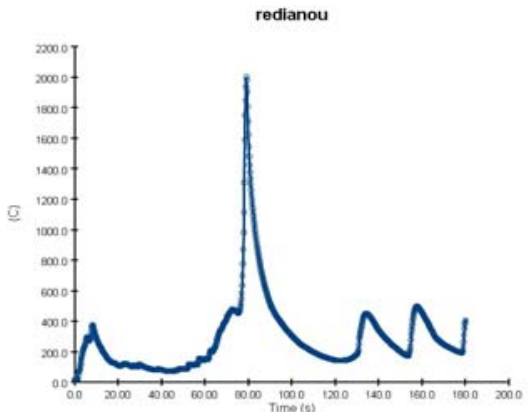

Figure 11 . The temperature changes detected by the thermocouple

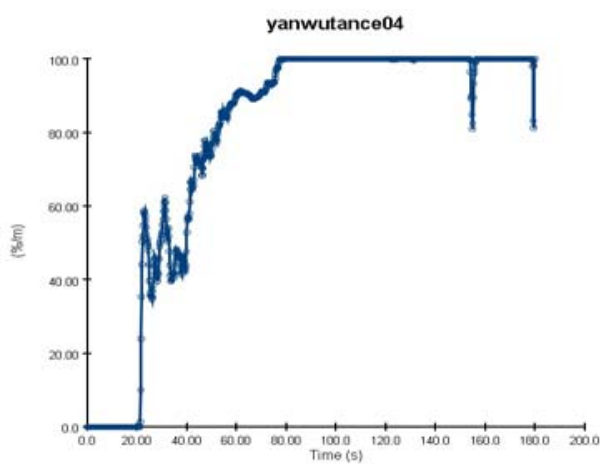

Fig.12 The smoke concentration detected by smoke detector

We can analyze the development of fire and the evacuation in different circumstances and also get the time of evacuation by changing the occupant density. Then we can obtain the function between the evacuation and the occupant density by data fitting, and then provide a scientific and reasonable proposal on fire and person evacuation to minimize the loss of fire. Besides that, we can develop virtual fire-fighting training system to provide real data of fire. Such as the fire development without human control, the amount of liquid sprayed to extinguish the fire and location of the fire suppression. So the virtual fire training system will offer the training and the simulation more like the real fire.

\section{SUMMARY}

This paper introduces a kind of computer simulation method for aircraft cabin fire and a fire dynamics simulation software. Details of the theoretical basis of FDS software and 
the methods and precautions are introduced, which offer a new method for the small probability event like airliner fire. The results obtained by computer simulation can apply to the actual fire rescue and evacuation operations, and are very useful to the practice.

Of course, the reliability of this method is also to be verified by practice. About FDS software itself, there are some shortcomings and deficiencies, such as the difficulty to establish the simulation model, and the model of complex shape may have a great errors etc., but we can believe that with the continuing improvement, this approach will be used a lot in the fire simulation.

\section{REFERENCES}

[1] Niu Xue-min, Xie Yong-qi,Yu Jian-zu. No ventilation helicopter power compartment fire temperature field numerical simulation [J]. Journal of Aerospace Power,2011,26 (8): 1761-1767

[2] Kevin McGrattan, Simo Hosikka. Fire Dynamic Simulator(Vision 5) Technical Reference Guide [M].USA: National Institute of Standards and Technology,2008:15-20

[3] Xu You-ping, Zhang Teng.The application of FDS in industry fire [J]. Industrial Safety and Environmental Protection, 2008,34 (5): 60-61

[4] Xing Zhixiang, Dai Chuang. The coliseum fire safety evacuation simulation[J]. Building Fire Protection Design, 2011, 30 (3): 201206

[5] Timo Korhonen,Simo Hostikka. Fire Dynamics Simulator with Evacuation:FDS+Evac Technical Reference and Use's Guide[M].VTT,2009 